\title{
Voice Controlled BOEbot using PointBug Algorithm for Human Robot Interaction using Android Technology
}

\author{
Nupur Choudhury \\ Computer Science and Engineering Department \\ Sikkim Manipal Institute of Technology \\ Sikkim, India
}

\author{
Chingtham Tejbanta Singh \\ Computer Science and Engineering Department \\ Sikkim Manipal Institute of Technology \\ Sikkim, India
}

\begin{abstract}
This paper deals with the research that led to the development of a robotic guide or a robot which would act as an aid for the aged category of the society. The robot is developed in such a way that it can be controlled and maneuvered by any device that is capable of supporting an android operating system. The user will be able to send the voice instructions using an android application which will be received via the Bluetooth module in the robots chassis and finally the instructions will be executed. The route map will be fed into the robot in the form of instructions that would follow a certain path and hence would be able to reach the target location detecting and avoiding all the obstacles through the infrared sensors that might be in the way to reach the destination. The project also gives an idea of the bug family algorithm, the PointBug algorithm that has been implemented for local path planning and obstacle detection and avoidance. The entire project is developed in such a way that the various compatibility constraints can be met with a wide variety of smart equipment that includes a smart phone, a tablet or a laptop etc. that holds the capability of supporting an android operating system in its architecture.
\end{abstract}

\section{Keywords}

BOEbot, infrared sensors, QTI sensors, route map, Google Voice to Text API, Speech Recognition, RFID, Bluetooth, PointBug Algorithm

\section{INTRODUCTION}

At present majority of the nations of the world are approaching the aged society at a very fast rate which leads to a lot of people and a huge part of the population to suffer from the limitations related to limb movement and deficiencies in motion and mobility which might be a result of the nerve related issues or lack of physical strength. Moreover the deficiencies related to assistance and nursing is an outcome of the increasing number of old aged people in the society. Therefore there is an urgent requirement of partial level replacement for the therapists and nurses via assistance guides. Presently numerous researchers are working on robot assistance which primarily concentrates on applications and projects related to limb movement and learning and training assistance for the human body system. The primary human activities includes locomotion or mobility, to enhance whose performance various walker types robots focusing on rehabilitation are being developed and is currently an encouraging and dominating field of research related to Human Robot Interaction. Currently various robotic frameworks that includes active and passive wheels and a walker type architecture are being deployed for assistive as well as ambulatory services providing satisfactory services to the potential users to witness cost effective satisfactory services. In addition to it majority of the research in Human Robot Interaction is centered round development of intelligent passive robotic walkers for the aged people of the society. However ongoing research activities. However the present researches are impacted with numerous loopholes and limitations primarily due to the following reasons:

- Most of the walker type robots that have developed are limited to indoor environments only which leads to the deficiency of maneuverability within indoor environments. Hence the mobility of the robot is limited to a fixed environmental domain.

- However the enormous size of the developed robotic guides also acts as a roadblock to a majority of the developed robotic guides as most of the environmental spaces consists of narrow spaces which behaves as a mobility restrictor in most of the case limiting the maneuverability due to its large size and unrestricted weight.

Hence in order to enable a higher quality life and accelerate the provision of assisting the aged society to move about freely in the outer world sufficient aid in the form of precise, easy handling and compatible devices are required. In order to facilitate this category of requirement an easy handling and simple robotic assistance guide has been developed which is confident of countering the above limitations and serve as an efficient mechanism to help the old people. The construction and the design of the robotic guide will consist of a BOEbot (Board of Education), a product of Parallax Inc. the design of the BOEbot will be such that it will be controlled using voice commands and also via manual control that will be incorporated over an android application. The android application will have 10 directional keys altogether and on pressing each key a specific set of instruction will be passed onto the robot which will make the robot move in the preprogrammed direction. These 10 directional keys would also work for speech related commands where the specific voice commands will be able to be transferred via Bluetooth to the robot's chassis and finally the instruction will be executed. The robot BOEbot is particularly chosen for this project because it overcomes the limitations and deficiencies that has been state before due to its light weight and small size and its easy maneuverability in outdoor environmental domain. The robot side coding is developed using BASIC stamp 2.5 editor which is easy to implement and can be implemented by naive developers. The robot brain consists of a microcontroller which need not be burnt with the program code but can simply be downloaded with the code and is extremely easy to decipher and execute the commands. Moreover the use of an android application in maneuvering the robot makes the entire application a user friendly and 
simple affair to be mounted on any device that is capable of supporting an android operating system components.

\section{BACKGROUND AND LITERATURE SURVEY}

Human Robot Interaction is the field of Computer Science oriented research domain which involves mechanisms like designing, understanding and evaluation of robot dependent and designed systems for the use of human beings. The communication that generates between the man and robot while following the above mechanisms can be classified into 2 primary categories:

- Proximate Interaction: This mode of communication deals with the human and robots that are collocated in time and space. For example the various service robots and assistive robots that are used in Human Robot Interaction generally located in the same time frame and space are classified in this category.

- Remote Interaction: Typically this type of interaction deals with the interaction between robots and humans that are not collocated in the similar time zone or spatial domain. For eg: Mars Rovers etc. which are deployed in the space and other planets to obtain information from.

Depending on the type of supervisory control, tele operations and social interaction the communication can be termed remote interactions. However physical interaction that might be classified into robot based assistance which involves mechanisms of interaction in the form of companions and peers are a part of proximate interaction. Several researches in the existing domain has created exceptional results in the field of Human Robot Interaction. O. Khatib et al., [1] have studied on the development of algorithms on models, strategies and algorithms that are primarily dependent on the various autonomous capabilities which aids Human Robot Interaction field. These researches are related to the manipulation and integrated mobility, interaction efficiency with the humans and cooperative skills between multiple robots. Hyun Keun Park et al. [4] developed a robot system named DO-U-MI to be used for nursing for The Elderly and The Disabled. The robot thus developed will help the old and handicapped people to move freely in the indoor environment primarily focusing on moving inside a nursing home. The project is extremely user friendly, however a minor limitation in case of manual control and lack of speech recognition comes into picture in this scenario. A major significant research work done by R. Rangarajan et al. [5], leads to the development and deployment of a speech controlled assistance robotic guide for the visually impaired so that it can aid the aged people as well as the blind people to reach the target location avoiding the various types of traffic and hindrances on the pathway. The robot can also charge on its own and can behave as a watchdog thereby proving an efficient mechanism for human robot interaction. Similar work has been done by Arpit Sharma et al. [7] and they have developed a robot which is arduino based and this robot can be controlled via an android phone wherein an android application has been developed in order to control the maneuverability of the robot. Related works are also being done in the field of automated mobility issues and orientation for the visually impaired people by Abdel Ilah Nour Alshbatat [8], which involves the implementation of GSM-GPS module to pin point the location of the individual as well as to provide any type of information that is related to the obstacles that are obtained from the ultrasonic sensors. However the system depend on braille learning for its operation due to which it might be problem for its use as the individuals need to be trained exclusively. However results have shown that the blind people can smoothly travel to their respective destinations with ease and comfort. A smart vision prototype for navigation has been developed by Joao Jose et al.[9] which comes in wearable form and is concise in shape and size. Efficient Human Robot Interaction is achieved wherein dynamic obstacles are being avoided and the main functionality involve a camera to be worn at the height of the chest and is connected to a laptop that is worn in a bag pack or a pouch. It also consists of a speaker into which the user can speak into so that effective interaction with the machine is obtained. Similarly Brice Burger et al. [10] studied on multimodal interaction between the human and robots specifically focusing on mutual assistance between speech and vision. A speech processing system was developed that detects the anaphoric and deictic utterances in speech forms. Life has become easier for the aged society with the various inventions and developments in the Human Robot Interaction field. Cumbersome activities like shopping etc. are also being dealt with in this research area wherein Chaitanya Gharpure et al. [11] developed mechanisms for enabling the blind people to move around for shopping experiences. This research has revealed several issues related to spatial cognition and product selectionin supermarkets. Additionally, Songmin Jia et al. worked on multiple user interfaces which has been deployed to enhance and improve quality of life, care and cost. Here an extensive use for the Radio Frequency Identification tags are being done which is easily detected by a camera and thus enables the user to avoid the obstacles. In a similar process Iwan Ulrich et al., [13], developed a Guide cane which could assist the elderly people to move about avoiding the obstacles as robotic technologies could be implemented by the device. However speech recognition and dynamic obstacles still are a limitation to this work.

\section{RESEARCH LIMITATIONS}

The various researches that has been carried out in this domain comprises of a large number of limitations which primarily are categorized into:

\subsection{Portability}

The various researches that are carried out in this domain consists of large number of equipment that are clubbed together for eg: laptops are used to maneuver the robots which results in the enormous size of the entire robotic system and hence would prove as a limitation for an efficient rehabilitation. The robot that has been developed would be able to overcome this type of limitation as it is smaller in size and can move about freely in narrow spaces which requires no additional heavy equipment to be carried around. It requires the basic utility mobile phone which can be carried out with ease as a daily utility device.

\subsection{Absence of Speech Recognition system}

The various robotic systems that are developed lacks in a speech recognition system through which the user can send voice commands and the robot can execute the instructions depending on the commands. The BOEbot that has been developed has Voice recognition module incorporated into the android application by adding Google Voice to Text application programmers interface along with the android application that has been developed. The voice commands would be sent using Bluetooth via an android phone and the instructions thus received would be executed with the 
instructions thus received overcoming the mentioned limitation.

\subsection{Lack of Route Decision System}

Currently most of the available models lacks in the procedures related to decision making as there are various loopholes as to which possible path the robot should take to reach the target faster avoiding the obstacles that might be present on the way. The BOEbot that has been developed addresses these kind of issues as it will be equipped with suitable path finding mechanisms as well as obstacle avoidance measures which makes the robot to move freely and reach the target location without colliding with any of the obstacles that might be on the way.

\subsection{Complex Operation Techniques}

There is an involvement of complex operation mechanisms in the existing systems which is a result of involvement of a large number of sensors and radio frequency identification tags thereby resulting in a complex framework which is cumbersome for use by a common and naïve user.

\subsection{Occurrence of Dynamic obstacles on the route}

It is widely observed that maximum of the robotic guides that has been developed lacks in an obstacle detection and avoidance mechanism which results in inefficient operations related to dynamic obstacles and to some extent a huge number of failures are a result of such a limitation. The BOEbot developed in this project can efficiently tackle the mentioned drawback leading to a better system with minimum failures.

\section{METHODOLOGY}

\subsection{Module 1: Development of an android application that controls a BOEbot}

The development process initially comprises of the design and development of an Android application through which the user can send the instructions in the form of keystrokes via the Bluetooth of the device. There are basically 10 keys which has a predefined key value that has been appended to it .On pressing of each key the associated key value will be transferred from the android application to the robots chassis and thereby execution of the instructions would follow. The Bluetooth device that has used in the architecture involves Easy Blue 500 a proprietary of Parallax Inc. The keys are forward, backward, left, right, lab1, lab2, OA round, stop respectively. Each key is responsible for sending its own key value to the robots chassis and in turn the robot carries out the required functionality. The application is built in such a way that the keystroke values could be transferred using the Bluetooth port of the device and can work towards the execution of those instructions.

\subsection{Module 2: Integration of speech recognition}

The second module is comprised of the integration of voice recognition module with module 1 which is done by integrating Google Voice to Text Application Programming Interface which enables the user to send the same voice commands through the phone and the robot can perform the necessary action regarding the commands that will be sent. At first the user will speak into the device and then the google API will transfer the speech into cloud and will go for a comparison for the matched text. The text thus retrieved will then be redirected back to the application and then a string match operation will follow. It needs to be mentioned that there are predefined strings and key values associated with it with. Post retrieval of the string the app then compares the string retrieved with the predefined string and the matched string value is then transferred to the robots chassis and the execution of the instructions would thus follow.

\subsection{Module 3: Obstacle Avoidance and path detection:}

The third module comprises of integration of the obstacle avoidance and path detection mechanisms to be implemented into the existing system. For obstacle avoidance, Infrared detectors shown in the figure 1 are being used along with Infrared receivers. The circuit diagram is as shown in figure: The infrared sensors thus integrated sends out IR signals to check out if there are any obstacles that might be on the way. The IR receivers in return reports back to the robots chassis with the result with values like 1 and 0 which would depict the presence of any obstacle in the path or not. The moment an obstacle is registered in the robots chassis the robot program in order to avoid the obstacle is executed which is to turn away from the obstacle and follow an entirely different route or to back off the main track until and unless the dynamic obstacle detected gets removed and the robot can move forward freely. The BOEbot thus developed follows a line as a part of the path planning activity where the robot will follow a line that has a darker colour as compared to its surroundings. For this purpose QTI sensors are used which are a different variety of infrared sensors that are capable of distinguishing between different shades or different colours. The QTI together with the infrared sensors will behave as the obstacle avoiding and path following mechanism through which the robot can reach the target location avoiding collision with any type of robots on the way and deciding on a particular route.

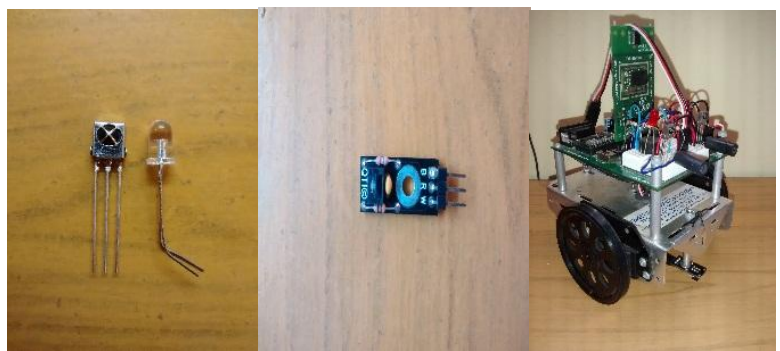

Figure 1: Images showing infrared sensors, QTI sensors, circuit for obstacle detection and avoidance.

\subsection{Module 4: Analysis of PointBug Algorithm for local path planning:}

One of the simplest path planning algorithm has been experimented with the current project for local path planning so that it can give an efficient path plan through which the BOEbot can navigate without colliding with the obstacles. PointBug algorithm that has been recently designed, and has the capability of navigating a robot's point in plane of environment which is unknown and is occupied with stationary and stagnant obstacles and objects of different shape. It usually calculates the next point to be moved from the current point so as to reach a particular point. The next point of the route is determined by the output of the range sensor which determines the distance from the sensor to the 
nearest obstacle. By considering a sharp change in the reading of the sensor output for increasing and decreasing distance the 'sudden change' in the distance from the sensor to the obstacle can be calculated. The difference $\Delta \mathrm{d}$ is defined for the distance which can be from infinity to a certain value or vice versa or between two values. Sudden point is calculated by the reading from the sensors within the time interval tn to $\mathrm{tn}+1$ which is utilized to detect different in range for $\Delta \mathrm{d}$. The robot will be able to survey the entire environment by rotating itself at a constant speed from $0^{0}$ to $360^{\circ}$. At the initial stage the robot faces the target and continuously rotates in left or right direction for the presence of any sudden points. On detection of the first sudden point, the rotation of the robot transforms to the position of the shortest or minimum straight line depicting the current point and target point found initially. The distance of the minimum measurement to the target is always recorded at a definite time interval. The sensor reading of the $180^{\circ}$ rotation is always ignored so that the previously recorded sudden points are not taken into consideration and duplicate results are not found. If the sensor does not gives any reading even after $360^{\circ}$ rotation then the robot assumes that there are no targets and it stops immediately.

The pseudo code of the algorithm is as follows:

While Not Target

If robot rotation $<=360$

Robot rotates right of left according to position of dmin

If sudden point

If 180 degree rotation

Ignore reading $/ *$ to avoid robot return to previous point */

Else

Get distance from current sudden point to next sudden point

Get angle of robot rotation

Move to new point according to distance and rotation angle

Record New dmin value

Reset rotation

End if

End if

Else

Robot Stop /* No sudden point and exit loop */

End if

While end

Robot Stop /* Robot successfully reaches target

The algorithm is expected operate to a certain extent in dynamic environment also as the information about the environment can be obtained gradually from the sensor readings during the maneuvering and movement of the robot. The performance of the algorithm of execution depends on total sudden points detected. The lesser the number of sudden points detection of the algorithm gets better. A simple maze representation of an environment is shown in fig 2(a). The sudden points would be generated utilizing the PointBug algorithm are also shown. The sudden points would be generated at every vertex of the rectangular block. In this process, the algorithm will generate the shortest route from starting point to target point as shown in figure 1(a).

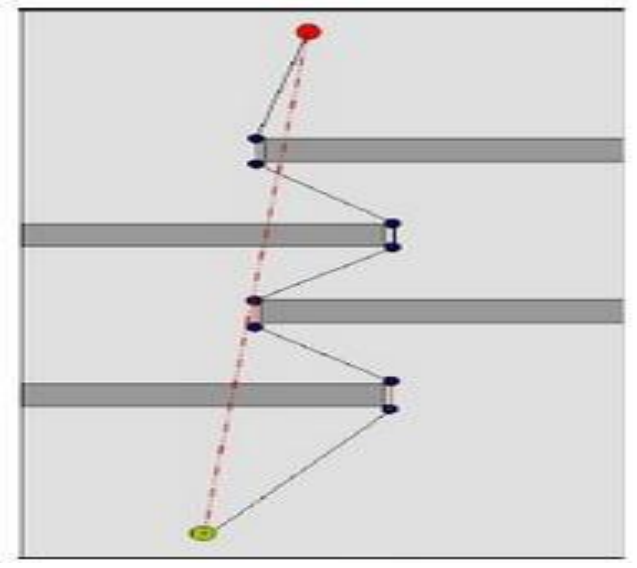

Fig. 1(a) sudden points and trajectory generated using PointBug Algorithm in simple maze based environment.

\subsection{Overall Architecture: The overall architecture of the system of the framework is shown in Fig 2.}

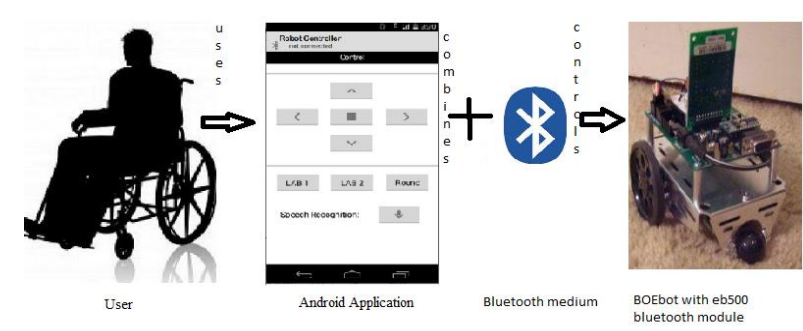

Fig 2: Framework Architecture

The overall architecture of the system consists of a human that would be operating on an android application which in turn would be sending instructions via the Bluetooth and which in turn would be received in the robot's chassis and make the robot to execute the commands that would be sent manually as well as via speech commands. The Bluetooth eb500 module is responsible for receiving the instructions and the program that has been loaded in the microcontroller Microchip PIC16C57c finally leads to the execution of the commands and makes the robot to carry out instructions like moving in various directions and carry out different functionalities like obstacle detection, avoidance etc.

\section{HARDWARE AND SOFTWARE REQUIREMENTS}

The hardware and software requirements of the entire project is given below

\section{Software requirements of the system}

- Eclipse IDE-Juno with the Android plugin, Java Developers version.

- Java Development Kit (JDK)

- Android SDK and add-ons

- Basic Stamp 2.5 editor (Parallax Inc.). 


\section{Hardware framework of the system}

- $\quad$ RAM 3 GB and above for high end processing of the android application

- (Board of Education BOE)bot (Parallax Inc.) robot.

- EasyBlue 500 SER Bluetooth module

- IR detectors, IR receivers, resistors(8-12 ohm) (Parallax Inc.)

- Q-Charge T-transfer I-infrared sensors, QTI receivers. (Parallax Inc.)

- Piazzio speaker (Parallax Inc.)

- Voltage Requirements 5- $15 \mathrm{~V}$ (DC) for Basic Stamp

- Current Draw@5 Volts 3 mA Run, for Basic Stamp

- Smart phone or a tablet capable of supporting an android operating system.

The primary development phase of the robot involves the development of an android application which can be mounted on a device that is capable of supporting an android application. The application is then connected to the robots chassis using the Bluetooth module. The brain of the robot constitutes a Basic stamp 2 microcontroller into which the program can simply be downloaded and can be executed. It is cost effective, easily available and can be advantageous as compared to any ARM based processing unit. The BOEbot and the EasyBlue Bluetooth module that has been used is as shown in the figures shown below.
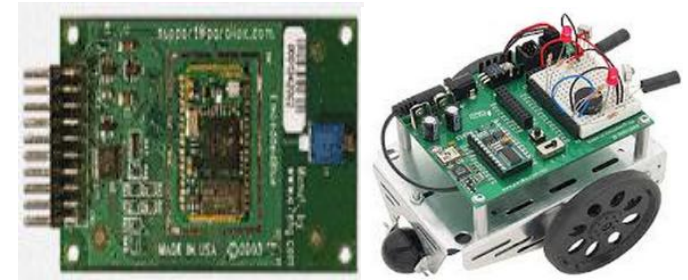

Fig 3: EasyBlue 500 SER module and a BOEbot

Java programming language along with Android SDK and add-ons are used for the development of the android application. The application development involves Eclipse IDE and Java Development Kit (JDK) and Basic Stamp 2-2.5 is .used to write into it. The android application looks like Fig 3 that is shown below:

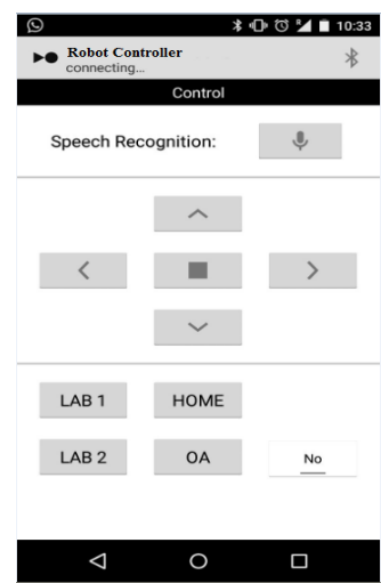

Fig 4: Android Application
The four different keys in the upper panel of the application will make the robot move in four different directions with involves left, right, front and backwards The five keys present in the lower panel of the application represents the functionality that involves the robot to maneuver to 2 predefined location for experimental analysis and then a take a round trip from the destination points. The OA tab is used for the conversion of the robot to obstacle detection and avoidance mode so that the robot can reach the target without any collision. This tab is also an implementation module of the PointBug algorithm for obstacle avoidance and detection. The Google voice to text API is used and is used for the recognition of voice commands thereby creating an interface as to where the user will speak and retrieve the voice commands in the form of text or strings which are then finally processed by string matching commands that are deployed in the application itself and the corresponding instruction after string match are sent to the target BOEbot's microcontroller to derive the necessary actions for the functionalities. This application can be supported in all the android operated devices.

\section{DESIGN}

The primary design of the system is categorized into 2 main frameworks:

\subsection{Wired framework design:}

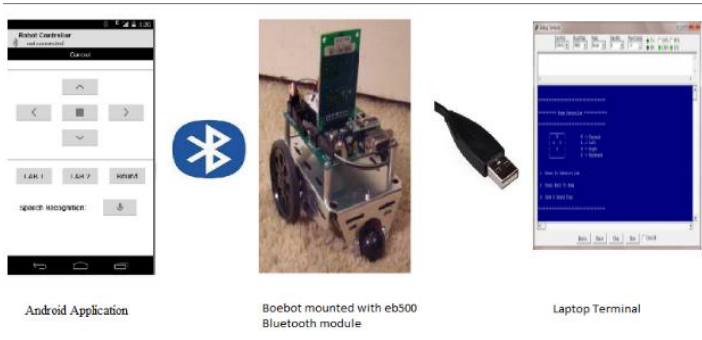

Fig 5: Wired framework design

The initial system design comprises of the BOEbot robot to be connected to a laptop via a USB cable and the program thus used to maneuver the robot is simply dumped into the microcontroller of the robot through the wired medium. The android application will be sending the keystrokes via the wireless medium i.e. the Bluetooth module that is being mounted on the robot will make the microcontroller receive the instructions.

\subsection{Wireless framework design:}
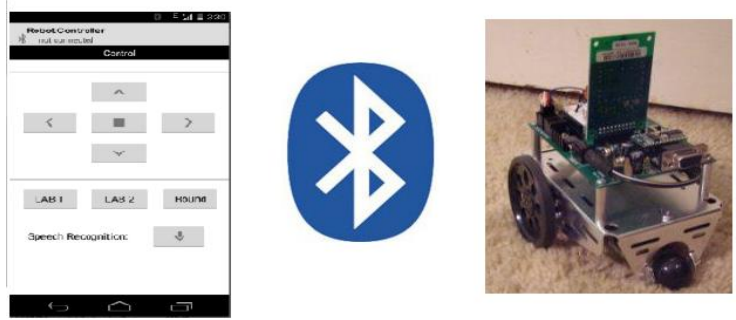

Android Application

Fig 6: Wireless communication design 
The final framework consists of wireless mode of communication from both the application and the robots end through which the voice commands and keystrokes can be transferred to the robots chassis and finally the robot executes the processes in a completely wireless manner.

\section{ACHIEVED GOAL}

The following are the primary functionalities that are executed by the developed project from the laptop terminal thus directing the robot to move in any particular direction.

- Forward, Backward, Left, Right with the help of the four keys. Of the keyboard W, A, S and D respectively.

- Steer to Lab 1 using LAB1 key

- $\quad$ Steer to Lab 2 using LAB2 key

- Steer to Home using Home key

- Execute obstacle avoidance mode using the OA tab which implements obstacle avoidance using PointBug algorithm.

The left, right, forward, backward keys will make the robot move to the respective directions till the key is continuously pressed. The Steer to Lab 1 and Steer to Lab 2 functionalities will be accomplished when the robot will move 22 seconds forward and then take a left turn or a right turn depending on LAB1 and LAB2 respectively. The HOME tab is responsible to move the robot back from the target location to the start point. The fifth functionality is used to implement the PointBug algorithm which will follow a particular line and then on encountering of an obstacle is capable of detecting it and follows the shortest distance to the target. These type of similar functionalities are also attained using the android application. However an additional benefit to it comes in the process when speech recognition was integrated with the current application and BOEbot can be controlled wirelessly through the android application on any android device by simply sending voice commands into the console. The app is capable of recognizing all of the above instructions and can perform similar functionalities with lesser amount of effort and manpower involved.

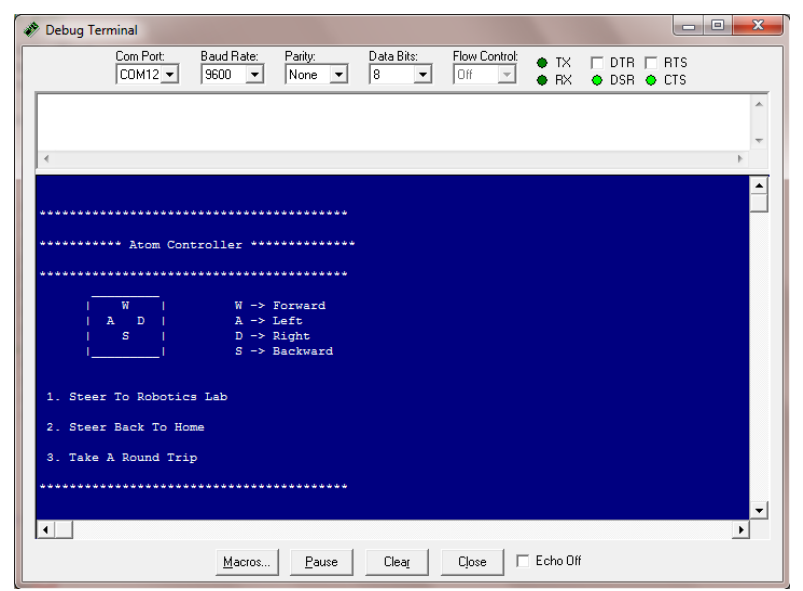

Fig 7: Laptop Implementation window which enable the BOEbot to be controlled via keyboard in a wired communication.

\section{CONCLUSION}

This project would be a boon to the research related to Human Robot Interaction domain and can assist the aged society and the partially visually impaired category of people to a great extent. Maneuverability, locomotion and mobility would become a simple affair in the presence of such type of human assistive guides which involves minimum effort in addition to keeping pace with the latest technology which behaves as a daily utility service in the present scenario. The application enormously reduces manual effort and no prior training is required to use this equipment. It will prove much more user friendly to the blind people as it can be controlled by voice commands and no prior knowledge about braille is required to efficiently use this application. The application is built using the latest technology and hence it is compatible with any type of devices that can support an android operating system proving it to be a blessing in technology to make life simpler

\section{REFERENCES}

[1] O. Khatib, K. Yokoi, O. Brock, K. Chang, and A. Casal,Robotics Laboratory,Department of Computer Science Stanford University, Stanford, California, Robots in Human Environments, [Available online: www.redaktion.tu-berlin.de , Last Access: October 2014].

[2] How Bluetooth Technology Works, [Available online: www.bluetooth.com/bluetooth/technology/works, Last Access: October 2014].

[3] Vladimir Kulyukin, Chaitanya Gharpure Nathan De Graw, Computer Science Department Utah State University Logan. Human-Robot Interaction in a Robotic Guide for the Visually Impaired,[Available online: www.aaai.org (American Association for Artificial Intelligence). Last Access: October 2014].

[4] Hyun Keun Park, Hyun Seok Hong, Han Jo Kwon, and Myung Jin Chung, Department of Electrical Engineering \& Computer Science Korea Advanced Institute of Science and Technology, A Nursing Robot System for The Elderly and The Disabled, [Available online: http://www.pdx.edu , Last Access: September 2014].

[5] R.Rangarajan, Mrs.B.Benslija M.E., Sri Muthukumaran Institute of Technology Chennai, Tamil Nadu, India, Voice Recognition Robotic Dog Guides For Visually Impaired People, IOSR Journal of Electronics and Communication Engineering (IOSR-JECE) e-ISSN: 2278-2834,p- ISSN: 2278-8735. Volume 9, Issue 2, PP 133-139, Ver. V ,Mar - Apr. 2014.

[6] M. Joshuva Regan, S.R.Barkunan, Anna University Regional Centre, Coimbatore, India, Voice Recognition Robot for Visually Impaired People, International Journal of Innovative Research in Computer and Communication Engineering, ISSN(Online): 2320-9801, Vol.2, Special Issue 1,March 2014.

[7] Arpit Sharma, Reetesh Verma, Saurabh Gupta and Sukhdeep Kaur Bhatia, Android Phone Controlled Robot Using Bluetooth, International Journal of Electronic and Electrical Engineering.ISSN 0974-2174, Volume 7, Number 5 (2014), pp. 443-448,2014.

[8] Abdel Ilah Nour Alshbatat, Department of Electrical Engineering, Tafila Technical University, Tafila 66110 , Jordan, Automated Mobility and Orientation System for 
Blind or Partially Sighted People, International Journal On Smart Sensing And Intelligent Systems, Vol. 6, no. 2, ISSN-11885608,April 2013.

[9] Joao Jose, Miguel Farrajota, João M.F. Rodrigues, J.M. Hans du Buf, Vision Laboratory, Institute for Systems and Robotics (ISR), University of the Algarve (FCT and ISE), Faro, Portugal,The Smart Vision local Navigation Aid for Blind and Visually Impaired Persons, International Journal of Digital Content Technology and its Applications Vol.5 No.5, May 2011, May 2011.

[10] Brice Burger, Frederic Lerasle, Isabelle Ferran, Aurelie Clodic, University de Toulouse, France, Mutual assistance between speech and vision for human-robot interaction, Intelligent Robots and Systems, 2008. IROS 2008. IEEE/RSJ International Conference on Sept, 2226, pp- 4011 - 4016 ,ISBN: 978-1-4244-2057-5 ,September 2008

[11] Chaitanya Gharpure Vladimir Kulyukin, Utah State University Logan,, Robot-Assisted Shopping for the Blind: Issues in Spatial Cognition and Product Selection .
Intelligent Service Robotics ,Springer-verlag 2008,eISSN: 1861-2776 ,p-ISSN: 1861-2784, Volume 1, Issue 3, pp 237-251, July 2008.

[12] Songmin Jia, Kunikatsu Takase University of ElectroCommunications, Tokyo Japan, 2008, Development of Service Robot System With Multiple Human User Interface, Human Robot Interaction by Nilanjan Sarkar, ISBN 978-3-902613-13-4,September 2007

[13] Iwan Ulrich and Johann Borenstein, Member, IEEE , The Guide Cane --Applying Mobile Robot Technologies to Assist the Visually Impaired ,Robotics \& Automation Magazine, IEEE ,Volume:10 , Issue: 1 ,ISSN-10709932,pp:9-20,March 2003

[14] O. Rogalla, M. Ehrenmann, R. Z"ollner, R. Becher and R. Dillmann,Using Gesture and Speech Control for Commanding a Robot Assistant, Robot and Human Interactive Communication, 2002. Proceedings. 11th IEEE International Workshop on 2002, ISBN :0-78037545-9, 2002 